\title{
Supersymmetric flat directions and resonant gravitino production
}

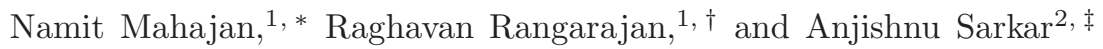 \\ ${ }^{1}$ Theoretical Physics Division, Physical Research Laboratory, Navrangpura, Ahmedabad 380 009, India \\ 2 LNM Institute of Information Technology, Jaipur 302031, India
}

(Dated: June 7, 2021)

\begin{abstract}
We study resonant gravitino production in the early Universe in the presence of SUSY flat directions whose large VEVs break some but not all gauge symmetries. We find that for a large region of parameter space the gravitino abundance is several orders of magnitude larger than the cosmological upper bound. Since flat directions with large VEVs are generically expected in supersymmetric theories this result further exacerbates the gravitino problem.
\end{abstract}

PACS numbers: 98.80.Cq,12.60.Jv

\section{INTRODUCTION}

Supersymmetric theories generically admit a large landscape of moduli space or flat directions along which the potential vanishes classically [1]. The flat directions can be described in terms of gauge invariant monomials that are built out of chiral superfields $\phi_{k}$ subject to specific constraints originating due to F- and D-flat requirements; in the context of the Minimal Supersymmetric Standard Model (MSSM), flat directions have been catalogued in [2, 3]. A flat direction can be represented by a modulus field $\phi$ and the different SUSY preserving vacua along the flat direction, i.e. different choices of the flat direction VEV, are not physically equivalent. Supersymmetry breaking lifts the flat directions, and in the early Universe $\phi$ can be displaced away from the origin with a large vacuum expectation value (VEV).

Non-zero VEVs for flat directions typically break one or more gauge symmetries, and the corresponding gauge supermultiplet acquires a mass $\propto \varphi$, where $\varphi$ is the VEV of $\phi$. Since $\varphi$ can be very large, scattering processes mediated by the heavy gauge bosons get suppressed, and the thermal history of the Universe can be very different from the standard thermal history of the Universe (see Ref. [4], and references therein). For example, if a flat direction associated with a squark field gets a VEV it breaks all gauge symmetries. This then leads to a delay in thermalization after inflation which can suppress gravitino production [5-7].

If, on the other hand, the flat direction under consideration preserves some of the gauge symmetries, then there can be reasonably fast thermalisation. In this article we suggest a new mechanism for enhanced gravitino production in the presence of a SUSY flat direction with a large $\mathrm{VEV}$ in the context of a thermal Universe. We find that in a large region of the parameter space the gravitino abundance is several orders of magnitude larger than the cosmological upper bound. For concreteness we

\footnotetext{
*Electronic address: nmahajan@prl.res.in

$\dagger$ Electronic address: raghavan@prl.res.in

‡Electronic address: anjishnu@lnmiit.ac.in
}

consider the specific flat direction $H_{u} H_{d}$. This breaks $S U(3)_{C} \times S U(2)_{L} \times U(1)_{Y} \rightarrow S U(3)_{C} \times U(1)_{\mathrm{EM}}$. This means that the gluon and gluino, and photon and photino do not get mass due to large $\varphi$ (allowing for thermalisation) while other particles coupled to the flat direction get a contribution to their mass proportional to $\varphi$. As we discuss below, there can be large resonant gravitino production in such a scenario when the intermediate particle in the s-channel process goes on the mass shell.

At finite temperature, the SUSY breaking scale, $M_{S}$, and the mass splitting between a particle and its superpartner, are set by the temperature $T$ of the thermal bath (see for example [8]). Then $M^{2}-m^{2}=\delta T^{2}+m_{0}^{2}$, where $\delta$ denotes the splitting due to the finite temperature between the square of the sfermion mass $M$ and the fermion mass $m$ in units of $T^{2}$, and $m_{0}$ is the zero temperature soft SUSY breaking parameter. In our scenario, the thermal splitting dominates over $m_{0}^{2}$.

We further assume that $\varphi \gg T$. Quarks and charged leptons and their superpartners get a contribution to their mass $\propto \varphi$. Of these particles, those with a small Yukawa coupling to the flat direction will still be relativistic while others will be heavy.

$W$ and $Z$ and their superpartners will be heavy due to their coupling with the flat direction. The photino and gluino get a mass $\propto T$ due to SUSY breaking as above. The gravitino is much lighter. The gravitino mass is $m_{\tilde{G}} \sim M_{S}^{2} / M_{P}=\left(\delta^{\prime} T^{2} / M_{P}\right)$ where $M_{P}=M_{P l} / \sqrt{8 \pi} \simeq$ $2.4 \times 10^{18} \mathrm{GeV}$ is the reduced Planck mass, and we take $\delta^{\prime} \sim 0.1$.

Now consider the following scattering reaction: $\tilde{A}+$ $f \longrightarrow \tilde{f}^{*} \longrightarrow f+\tilde{G}$, where $f, \tilde{A}, \tilde{G}, \tilde{f}$ denote a charged (heavy) fermion, gluino/photino, gravitino and sfermion respectively. (For example, one could consider quark and gluino scattering to quark and gravitino.) Since $\varphi \gg T \gg m_{0}$ implying $\delta T^{2}, m_{0}^{2} \ll m^{2}$, the initial state fermion and intermediate sfermion are almost degenerate in mass. Moreover, the sfermion in the s-channel exchange can be on the mass shell. This Breit-Wigner resonance then gives a large contribution to the scattering cross section and a very large abundance of gravitinos. The Boltzmann suppression of the incoming heavy fermion is compensated by the Breit-Wigner resonance 
factor.

Neglecting other contributions, the s-channel resonant production cross section is given by (considering only the helcity $1 / 2$ component for the gravitino)

$$
\sigma(s) \approx \frac{1}{3.8 .2 .2} \frac{2 N_{g}}{4} \frac{\left(M^{2}-m^{2}\right)^{2}}{3 m_{\tilde{G}}^{2} M_{P}^{2}} \frac{\alpha_{g}}{s} \frac{\left(s-m^{2}\right)^{2}}{\left.\left(s-M^{2}\right)^{2}+M^{2}{ }^{2}\right)}
$$

where $\Gamma \ll M$ is the width of the intermediate on-shell sfermion, $\alpha_{g}=g^{2} /(4 \pi)$ where $g$ is the relevant gauge coupling, and we have used $m_{\tilde{G}} \ll m, M$. Feynman rules for gravitino interacations are in Ref. [9], and $N_{g}$ is $\sum_{A} \operatorname{Tr}\left[T^{A} T^{A}\right]$ where $T^{A}$ is the generator of the relevant gauge group. Since we are considering the goldstino part of the gravitino, which comes from SUSY breaking, the cross section should not be $M_{P}$ suppressed (see for example [10]). Using $m_{\widetilde{G}} M_{P}=\delta^{\prime} T^{2}$ and $\left(M^{2}-m^{2}\right)=\delta T^{2}$ and assuming $\delta \sim \delta^{\prime}$ we get

$$
\sigma(s) \approx \frac{N_{g}}{576} \frac{\alpha_{g}}{s} \frac{\left(s-m^{2}\right)^{2}}{\left(s-M^{2}\right)^{2}+M^{2} \Gamma^{2}}
$$

We let $\Gamma=M / z$ and take $z=50,500$ in our analysis (though representative values for our scenario below may be much smaller). Lower values of $\Gamma$ can increase $\sigma$.

This should be contrasted with the zero temperature case, say within gravity mediated scenario where $M^{2}-m^{2} \sim m_{0}^{2} \ll m_{\widetilde{G}} M_{P}=M_{S}^{2}$ leading to a strong suppression factor. We emphasize again that this new and novel feature is due to distinctive character of supersymmetric theories at finite temperature.

The mechanism considered above is different from enhanced gravitino production during preheating which has been considered in Ref. [11 19].

\section{BOLTZMANN EQUATION}

Gravitinos are produced by the scattering of the decay products of the inflaton [20 39]. Refs. 24, 30] provide a list of processes for gravitino production in the standard scenario for thermal gravitino production.

The number density of a species $X_{3}$ participating in reactions $X_{1} X_{2} \rightleftharpoons X_{3} X_{4}$ can be obtained via the integrated Boltzmann equation,

$$
\dot{n}_{3}+3 H n_{3}=\mathcal{C}
$$

where $\mathcal{C}$ is the collision integral. When the number density of $X_{3}$ is small, as we presume in our case where $X_{3}$ represents the gravitino, we can ignore the $X_{3} X_{4} \rightarrow$ $X_{1} X_{2}$ process. Then

$$
\dot{n}_{3}+3 H n_{3}=\int d \Pi_{1} d \Pi_{2} f_{1} f_{2} W_{12}(s) \equiv A,
$$

where $f_{i}$ are phase space distribution functions and $d \Pi_{i} \equiv \frac{g_{i}}{(2 \pi)^{3}} \frac{d^{3} p_{i}}{2 E_{i}} . \quad g_{i}$ is the number of internal degrees of freedom of species $i$. Then, from Ref. [40],

$$
A=\frac{T}{32 \pi^{4}} \sum_{1,2} \int d s g_{1} g_{2} p_{12} W_{12} K_{1}\left(\frac{\sqrt{s}}{T}\right),
$$

where $W_{12}(s)=4 p_{12} \sqrt{s} \sigma_{C M}(s), \sigma_{C M}$ is the cross section in the centre-of-mass frame and

$$
p_{12}=\frac{\left[s-\left(m_{1}+m_{2}\right)^{2}\right]^{1 / 2}\left[s-\left(m_{1}-m_{2}\right)^{2}\right]^{1 / 2}}{2 \sqrt{s}}
$$

is the magnitude of the momentum of particle $X_{1}$ (or $X_{2}$ ) in the center-of-mass frame of the particle pair $\left(X_{1}, X_{2}\right)$. $K_{1}$ is the modified Bessel function of the second kind of order 1. Note that its exponential decay at large $s$ provides the Boltzmann suppression associated with the incoming heavy quark. ${ }^{1}$ For $p_{12}$, we get $\left(s-m^{2}\right) /(2 \sqrt{s})$ for the incoming gaugino mass smaller than $T$ and hence much smaller than $m$.

Substituting $\sigma_{C M}$ in $A$, we need to do the integral over $s$. We shall specifically consider the process

$$
\tilde{g}+q \longrightarrow \tilde{q}^{*} \longrightarrow q+\tilde{G} .
$$

For this process $g_{1}=2 \times 8$ and $g_{2}=2 \times 3$ and $\alpha_{g}$ is replaced by $\alpha_{s}$. Throughout we shall ignore the variation of $\alpha_{s}$ with temperature and take $\alpha_{s}=5 \times 10^{-2}$, as relevant for the temperatures in our scenario.

\section{RESONANT GRAVITINO PRODUCTION}

For obtaining $A$ we first discuss the evolution of $M \sim$ $m=h \varphi$, where $h$ is a relevant Yukawa coupling. ${ }^{2} \mathrm{We}$ take the mass of the flat direction $m_{\phi}$ to be related to the scale of SUSY breaking. Immediately after inflation, when the Universe is cold, $m_{\phi}=m_{0}$. When $H$ decreases to $H \sim m_{\phi}=m_{0}$ at $t_{0} \sim 1 / m_{0}, \phi$ starts oscillating and thereafter $\varphi$ decreases as $1 / a^{3 / 2}$. ( $a$ is the scale factor of the Universe.) Subsequently at $t_{d}$ the inflaton decays, the temperature becomes $T_{R}$ (in the instantaneous decay approximation) and then the temperature also determines the SUSY breaking scale and the mass of the flat direction: $m_{\phi}^{2}=h^{\prime 2} T^{2}+m_{0}^{2}$, where $h^{\prime}$ is the Yukawa coupling for some light field in thermal equilibrium. ${ }^{3} H=10 T^{2} / M_{P l}<m_{\phi}$ and $\phi$ continues to

1 The derivation of $A$ presumes a Maxwell-Boltzmann distribution for both incoming particles, while our gluino is relativistic. However it has been argued in Ref. [41] that final abundances are insensitive to the statistics.

We ignore gravitino decay in the Boltzmann equation as the gravitino lifetime is $10^{7-8}\left(100 \mathrm{GeV} / m_{\widetilde{G}}\right) \mathrm{s}$ [24] and is not relevant during the gravitino production era.

2 When $\phi$ is oscillating, $\varphi$ is the amplitude of oscillation, as the period of the oscillation $m_{\phi}^{-1}$ is much smaller than the timescale for gravitino production.

3 Thermal corrections to the flat direction potential of the form $h^{\prime 2} T^{2}|\phi|^{2}$ and $\alpha_{g}^{2} T^{4} \log \left(|\phi|^{2}\right)$, along with non-renormalisable 
oscillate after $t_{d}$. The oscillating field can be thought of as a condensate of zero momentum particles.

We take the initial VEV of $\phi$ at $t_{0}$ to be $\varphi_{0}$. Then for $t>t_{d}=\Gamma_{d}^{-1}$, where $\Gamma_{d}$ is the inflaton decay rate, the quark mass is given by

$$
m^{2}=h^{2} \varphi_{0}^{2}\left(\frac{a_{0}}{a_{d}}\right)^{3}\left(\frac{a_{d}}{a}\right)^{3}=h^{2} \varphi_{0}^{2}\left(\frac{\Gamma_{d}}{m_{0}}\right)^{2}\left(\frac{T}{T_{R}}\right)^{3}
$$

where we have used $a \sim t^{2 / 3}$ for $t_{0}<t<t_{d}$ for an inflaton oscillating in a quadratic potential during reheating and $a \sim 1 / T$ for $t>t_{d} . T_{R}$ is the reheat temperature at $t_{d}$ and is given by 45 .

$$
T_{R}=0.55 g_{* *}^{-1 / 4} \Gamma_{d}^{1 / 2} M_{\mathrm{Pl}}^{1 / 2}
$$

where $g_{* *}$ is the number of relativistic degrees of freedom relevant when the flat direction VEV is large and many species are non-relativistic. Taking the relativistic species to be the photino, photon, gluino and gluon, $g_{* *}=33.75$. We further define $m_{d} \equiv m\left(t_{d}\right)=$ $m_{t 0}\left(\Gamma_{d} / m_{0}\right)$, where $m_{t 0}=h \varphi_{0}$.

After the inflaton decays, the energy density $\rho_{\phi}$ in the flat direction condensate is $\frac{1}{2} m_{\phi}^{2} \varphi^{2}$ while the energy density of the radiation $\rho_{\text {rad }}=\left(\pi^{2} / 30\right) g_{* *} T^{4}$. For the parameter values we consider below, the Universe is radiation dominated after inflaton decay, and therefore

$$
T=T_{R}\left(\frac{t_{d}}{t}\right)^{\frac{1}{2}}
$$

$A$ in Eq. (5) is a function of $T$. $A$ can now be expressed as a function of $t$ and we can solve Eq. (4) to obtain the number density of gravitinos. We will finally like to obtain the gravitino number density at $t_{e}$ when the flat direction condensate decays, or the resonant mechanism terminates.

The condensate decays (perturbatively) at $t_{f}$ when its decay rate $\Gamma_{\phi}=m_{\phi}^{3} / \varphi^{2}$ equals $H$ [46, 47]. (We discuss alternate mechanisms for condensate decay below.) Then at any temperature, for $m_{\phi} \sim h^{\prime} T$

$$
\Gamma_{\phi}=h^{\prime 3} T^{3} / \varphi^{2}=h^{\prime 3} T^{3} /\left[\varphi_{d}^{2} T^{3} / T_{R}^{3}\right]=h^{\prime 3} T_{R}^{3} / \varphi_{d}^{2}
$$

using $\varphi^{2} \sim 1 / a^{3} \sim T^{3}$. Now $\varphi_{d}^{2}=\varphi_{0}^{2}\left(a_{0} / a_{d}\right)^{3}=$ $\varphi_{0}^{2}\left(t_{0} / t_{d}\right)^{2}=\varphi_{0}^{2}\left(\Gamma_{d} / m_{0}\right)^{2}$. Then,

$$
t_{f}=\frac{\varphi_{0}^{2} \Gamma_{d}^{2}}{h^{\prime 3} T_{R}^{3} m_{0}^{2}} .
$$

Let $T_{m}$ be the temperature when the condensate thermal mass $h^{\prime} T$ equals $m_{0}$. If $t_{f}$ obtained above is greater

terms, have been considered in Refs. [42 44]. We presume that thermal corrections to the flat direction potential is effectively quadratic with a contribution of $h^{\prime} T$ to the mass. For the light field with Yukawa coupling $h^{\prime}$ to be relativistic and in thermal equilibrium, its mass $h^{\prime} \varphi(t)$ should be less than $T(t)$. than $t_{m}=t_{d}\left(T_{R} / T_{m}\right)^{2}$, then one should use $m_{\phi}=m_{0}$ to obtain $t_{f} \cdot t_{f}$ is then obtained as in Eq. (46) of Ref. [7] as

$$
t_{f}=\frac{\varphi_{0}^{4 / 5} \Gamma_{d}^{1 / 5}}{m_{0}^{2}}
$$

In our numerical analysis below the thermal mass $h^{\prime} T$ for $\phi$ is less than $m_{0}$ at $t_{d}$ itself for the low reheat temperature that we consider.

It may happen that the resonant phenomena breaks down before $t_{f}$ at some time $t_{r}$. We require the sfermion and fermion masses to be much larger than $T$. Now the quark mass $\sim T^{3 / 2}$ and so falls faster than the temperature. Defining $T_{r}$ via $m\left(T_{r}\right)=T_{r}$ and using Eq. (8) we get

$$
T_{r}=\left(\frac{m_{0}}{\Gamma_{d}}\right)^{2}\left(\frac{T_{R}}{m_{t 0}}\right)^{2} T_{R}=\left(\frac{T_{R}}{m_{d}}\right)^{2} T_{R}
$$

As $t \sim a^{2} \sim 1 / T^{2}$ for $t>t_{d}$

$$
t_{r}=\left(\frac{m_{d}}{T_{R}}\right)^{4} t_{d}
$$

The final gravitino abundance is the abundance at $t_{e}=$ $\min \left(t_{f}, t_{r}\right)$ when resonant gravitino production ends and is given by

$$
Y\left(t_{e}\right) \equiv \frac{n\left(t_{e}\right)}{s\left(t_{e}\right)}
$$

where $s$ is the entropy density. We obtain the gravitino number density by solving the integrated Boltzmann equation till $t_{e}$. Now the temperature at $t_{e}$ just after the resonant gravitino production ends is $T_{e}^{\prime}=\left(g_{* *} / g_{*}\right)^{1 / 4} T_{e}$, where $T_{e}=T_{R}\left(t_{d} / t_{e}\right)^{1 / 2}$ is the temperature just before the end of resonant gravitino production. Then the entropy density is $s\left(t_{e}\right)=\left(2 \pi^{2} / 45\right) g_{*} T_{e}^{\prime 3}$ We take $g_{*}=228.75$. Note that the energy density in $\phi$ is subdominant when the flat direction decays for the cases considered below.

After the flat direction condensate decays the gravitino mass is given by the expression relevant to the mechanism of supersymmetry breaking. In gravity mediated supersymmetry breaking, $m_{3 / 2} \sim m_{0} \sim 100-1000 \mathrm{GeV}$. The abundance obtained above can then be compared with the corresponding upper limit of $10^{-14}$ obtained in Ref. [4] from various cosmological constraints for $m_{3 / 2} \sim 100 \mathrm{GeV}$. For $m_{3 / 2} \sim 1000 \mathrm{GeV}$ the upper limit is $10^{-16}$.

In addition to resonant gravitino production involving heavy quarks and squarks, gravitinos are also produced by the usual non-resonant thermal scattering of relativistic particles during reheating [33, 34, 36 39] and after reheating. The total abundance generated will be proportional to $T_{R}$. We shall choose $\Gamma_{d}$ such that the reheat temperature is low enough $\left(\leq 10^{6} \mathrm{GeV}\right)$ to suppress gravitino production via non-resonant thermal production. 


\section{RESULTS}

We now consider plausible values of $\varphi_{0}$. The non-zero vacuum energy during inflation breaks SUSY and can give large positive masses of order $H_{I}$ to the flat direction, where $H_{I}$ is the Hubble parameter during inflation [49, 50]. Then quantum fluctuations during inflation give a VEV of order $H_{I}$ [49]. Assuming that the field does not vary much till $t_{0}, \varphi_{0} \sim H_{I} \leq 10^{13} \mathrm{GeV}$.

Alternatively, in some theories the contribution to the flat direction potential during inflation due to $H_{I}$ is negative at the origin [49, 50]. This correction to the potential, along with non-renomalizable terms, leads to a shifted minimum of the potential. Then one obtains a large VEV of order $M_{P l}$ 50], or $10^{12-14} \mathrm{GeV}$ on including GUT interactions 51]. The shifted minimum of the potential for $\phi$ is $\Lambda(H / \Lambda)^{1 /(n+1)}$ for non-renormalizable terms of the form $\phi^{2 n+4} / \Lambda^{2 n}, n \geq 1$, where $\Lambda$ is the scale of some new physics [51, 52]. $\phi$ oscillates about this time dependent minimum which decreases as $H$ decreases. When $H \sim m_{0}$ at $t_{0}$, the potential minimum goes to zero and the field oscillates about the origin in a quadratic potential with curvature $m_{0}^{2}$. Then $\varphi_{0} \sim \Lambda\left(H\left(t_{0}\right) / \Lambda\right)^{1 /(n+1)}$, where $H\left(t_{0}\right)=m_{0}$ [52]. If $\Lambda \sim 10^{16} \mathrm{GeV}$, or $M_{\mathrm{Pl}}$, then we get $\varphi_{0} \sim 10^{9} \mathrm{GeV}$, or $3 \times 10^{10} \mathrm{GeV}$, for $n=1$. For larger $n, \varphi_{0}$ will be larger.

For our analysis below we present a few cases with different parameter values. We take $\Gamma_{d}=10^{-6} \mathrm{GeV}$ to ensure that the reheat temperature of $8 \times 10^{5} \mathrm{GeV}$ is below the upper bound of $10^{6} \mathrm{GeV}$ for (non-resonant) thermal gravitino production [53]. $t_{d}$ is then $10^{6} \mathrm{GeV}^{-1}$.

For $\varphi_{0}=10^{16} \mathrm{GeV}$ we take $z=50, \delta=0.1, h=0.5$, $h^{\prime}=10^{-5}$ and $m_{0}=100 \mathrm{GeV}$. For $\varphi_{0}=10^{17} \mathrm{GeV}$ we take $z=500, \delta=0.1, h^{\prime}=10^{-5}, m_{0}=100 \mathrm{GeV}$ with $h=0.1$ and $m_{0}=1000 \mathrm{GeV}$ with $h=0.2$. For the first case we obtain $Y=1 \times 10^{-5}$ at $t_{e}=4 \times 10^{7} \mathrm{GeV}^{-1}$. For the second case we obtain $Y=3 \times 10^{-8}$ at $t_{e}=$ $3 \times 10^{8} \mathrm{GeV}^{-1}$. For the third case we obtain $Y=7 \times 10^{-4}$ at $t_{e}=3 \times 10^{6} \mathrm{GeV}^{-1}$. All these abundances are much larger than the cosmological upper bounds of $10^{-14,-16}$ mentioned above.

We further point out that for $\varphi_{0} \leq 10^{15} \mathrm{GeV}$ the collision integral on the r.h.s. of the integrated Boltzmann equation is so large that one gets an abundance much larger than 1. While this is in conflict with the assumption of a small gravitino abundance presumed while obtaining Eq. (4), it indicates that the gravitino number density would be equal to the equilibrium gravitino number density in such cases. The abundance at $t_{e}$ is then $Y\left(t_{e}\right)=n_{\widetilde{G}}^{e q}\left(t_{e}\right) / s\left(t_{e}\right) \approx 8 \times 10^{-3}$, where the equilibrium gravitino number density $n_{\widetilde{G}}^{e q}\left(t_{e}\right)=3 \zeta(3) /\left(4 \pi^{2}\right) 2 T_{e}^{3}$.

The gravitino abundance is larger for smaller $m_{d}$ and larger $\delta$ as they increase the phase space available for resonant production. Because $m$ and $M$ are very close in mass, $M-m \approx\left(\delta T^{2}+m_{0}^{2}\right) /(2 m) \ll \Gamma / 2$, the initial value of $\sqrt{s}$ lies within the Breit-Wigner peak in the integral over $s$ in the cross section. Increasing $\delta$, or decreasing $m_{d}$, allows one to sample more of the Breit-Wigner resonance and thus gives a larger contribution. $m_{d}$ is a function of $h, \varphi_{0}, \Gamma_{d}$, and $m_{0}$. Decreasing $h, \varphi_{0}$ or $\Gamma_{d}$, or increasing $m_{0}$ (which makes the condensate oscillate earlier), decreases $m_{d}$ and increases the gravitino abundance. At later times one samples more of the Breit-Wigner resonance as $\delta T^{2} \sim 1 / a^{2}$ while $m \Gamma \sim 1 / a^{3}$.

Even though $A$ contains a Boltzmann suppression factor because of the heavy incoming quark, the resonance effect overcomes this suppression, as mentioned earlier. We have verified that for incoming energies away from resonance the gravitino production cross section is indeed suppressed.

We have only considered one channel for gravitino production for this flat direction. One can consider processes involving other particles such as photinos and charged leptons. Other flat directions with large VEVs can also lead to resonant gravitino production. For example, the flat direction parametrised by the monomial LLe will break $S U(2)_{L} \times U(1)_{Y}$. Gluons and gluinos could then participate in resonant gravitino production as above.

\section{DISCUSSION AND CONCLUSION}

Our results indicate that there can be large gravitino production through a resonant process in a thermal Universe in the presence of a large VEV for a SUSY flat direction that breaks some but not all gauge symmetries. For the parameters considered in the previous section we find that the gravitino abundance exceeds the cosmological upper bounds, and in many cases can equal the large equilibrium abundance. Since large VEVs for SUSY flat directions is a generic feature in supersymmetric cosmological scenarios our results are very relevant to the understanding of the gravitino problem in the early Universe.

Lowering the reheat temperature (by decreasing $\Gamma_{d}$ ) increases the gravitino abundance. This is in contrast with the standard non-resonant thermal production scenario in which the abundance is proportional to the reheat temperature. This implies that if we consider lowering the reheat temperature, the standard solution to the gravitino problem, it will lead to even more gravitino production from the resonant scattering process discussed above.

One mechanism to decrease the large gravitino abundance obtained above is to invoke the quick decay of the flat direction. The longevity of flat directions has been debated in Refs. 47, 54 62]. However it has been argued in Refs. [59, 62] that even if non-perturbative rapid decay via parametric resonance occurs for scenarios with multiple flat directions it leads to a redistribution of energy of the condensate amongst the fields in the D flat superspace and hence to practically the same cosmological consequences, including at least as large masses as in the scenario with only perturbative decay.

Scattering of particles of the thermal bath off the flat direction condensate can lead to the decay of the con- 
densate [2, 42, 43], though thermal effects are less important for larger values of $n$. For example, for $n=3$ the condensate decays much after the decay of the inflaton [43]. Decay via fragmentation into solitonic states called Q-balls 63 71] or Q-axitons [72] due to inhomogeneities in the condensate may also be relevant. The relevant time scale for Q-ball and Q-axiton formation is $10^{2-4} m^{-1}$, where $m$ is a mass scale associated with the flat direction 63, 64, 68, 72], which can decrease the lifetime of the flat directions considerably to even less than $t_{d}$. However, Q-balls/axitons may not form if there is no related conserved charge associated with the flat direc- tion (usually baryon or lepton number).

In conclusion, we have pointed out that there could be excessive gravitino production in the early Universe through a resonant mechanism in the presence of flat directions in supersymmetric theories. The final abundance can exceed the cosmological bound on the gravitino abundance by several orders of magnitude. This result would be relevant for typical supersymmetric scenarios of the early Universe, and exacerbates the well known gravitino problem. Mechanisms for the quick decay of the flat directions may need to be invoked to suppress the final gravitino abundance.
[1] I. Affleck, M. Dine and N. Seiberg, Nucl. Phys. B 241, 493 (1984).

[2] M. Dine, L. Randall and S. D. Thomas, Nucl. Phys. B 458, 291 (1996) hep-ph/9507453.

[3] T. Gherghetta, C. F. Kolda and S. P. Martin, Nucl. Phys. B 468, 37 (1996) hep-ph/9510370.

[4] K. Enqvist and A. Mazumdar, Phys. Rept. 380, 99 (2003) hep-ph/0209244.

[5] R. Allahverdi and A. Mazumdar, hep-ph/0505050

[6] R. Allahverdi and A. Mazumdar, JCAP 0610, 008 (2006) hep-ph/0512227.

[7] R. Rangarajan and A. Sarkar, Astropart. Phys. 48, 37 (2013) arXiv:1205.5408 [astro-ph.CO]].

[8] L. Girardello, M. T. Grisaru and P. Salomonson, Nucl. Phys. B 178, 331 (1981).

[9] T. Moroi, hep-ph/9503210 (see Fig. 4.4).

[10] S. P. Martin, hep-ph/9709356v6, see Sec. 7.5.

[11] G. F. Giudice, A. Riotto and I. Tkachev, JHEP 9911, 036 (1999) arXiv:hep-ph/9911302.

[12] A. L. Maroto and A. Mazumdar, Phys. Rev. Lett. 84, 1655 (2000) arXiv:hep-ph/9904206.

[13] R. Kallosh, L. Kofman, A. D. Linde and A. Van Proeyen, Phys. Rev. D 61, 103503 (2000) arXiv:hep-th/9907124.

[14] S. Tsujikawa, B. A. Bassett and F. Viniegra, JHEP 0008, 019 (2000) arXiv:hep-ph/0006354.

[15] H. P. Nilles, M. Peloso and L. Sorbo, Phys. Rev. Lett. 87, 051302 (2001) arXiv:hep-ph/0102264.

[16] H. P. Nilles, M. Peloso and L. Sorbo, JHEP 0104, 004 (2001) arXiv:hep-th/0103202.

[17] H. P. Nilles, K. A. Olive and M. Peloso, Phys. Lett. B 522, 304 (2001) arXiv:hep-ph/0107212.

[18] P. B. Greene, K. Kadota and H. Murayama, Phys. Rev. D 68, 043502 (2003) arXiv:hep-ph/0208276.

[19] D. I. Podolsky, G. N. Felder, L. Kofman and M. Peloso, Phys. Rev. D 73, 023501 (2006) arXiv:hep-ph/0507096.

[20] D. V. Nanopoulos, K. A. Olive and M. Srednicki, Phys. Lett. B 127, 30 (1983).

[21] L. M. Krauss, Nucl. Phys. B 227, 556 (1983).

[22] I. V. Falomkin, G. B. Pontecorvo, M. G. Sapozhnikov, M. Y. Khlopov, F. Balestra and G. Piragino, Nuovo Cim. A 79, 193 (1984) [Yad. Fiz. 39, 990 (1984)].

[23] M. Y. .Khlopov and A. D. Linde, Phys. Lett. B 138, 265 (1984).

[24] J. R. Ellis, J. E. Kim and D. V. Nanopoulos, Phys. Lett. B 145, 181 (1984).

[25] R. Juszkiewicz, J. Silk and A. Stebbins, Phys. Lett. B
158, 463 (1985)

[26] J. R. Ellis, D. V. Nanopoulos and S. Sarkar, Nucl. Phys. B 259, 175 (1985).

[27] M. Kawasaki and K. Sato, Phys. Lett. B 189, 23 (1987).

[28] M. Y. .Khlopov, Y. .L. Levitan, E. V. Sedelnikov and I. M. Sobol, Phys. Atom. Nucl. 57, 1393 (1994) [Yad. Fiz. 57, 1466 (1994)].

[29] T. Moroi, H. Murayama and M. Yamaguchi, Phys. Lett. B 303, 289 (1993).

[30] M. Kawasaki and T. Moroi, Prog. Theor. Phys. 93, 879 (1995) hep-ph/9403364, hep-ph/9403061.

[31] M. Bolz, A. Brandenburg and W. Buchmuller, Nucl. Phys. B 606, 518 (2001) [Erratum-ibid. B 790, 336 (2008)] hep-ph/0012052.

[32] R. H. Cyburt, J. R. Ellis, B. D. Fields and K. A. Olive, Phys. Rev. D 67, 103521 (2003) astro-ph/0211258.

[33] G. F. Giudice, A. Riotto and I. Tkachev, JHEP 9911, 036 (1999) hep-ph/9911302.

[34] M. Kawasaki, K. Kohri and T. Moroi, Phys. Rev. D 71, 083502 (2005) astro-ph/0408426.

[35] J. Pradler and F. D. Steffen, Phys. Rev. D 75, 023509 (2007) hep-ph/0608344.

[36] J. Pradler and F. D. Steffen, Phys. Lett. B 648, 224 (2007) hep-ph/0612291.

[37] R. Rangarajan and N. Sahu, Mod. Phys. Lett. A 23, 427 (2008) hep-ph/0606228.

[38] R. Rangarajan and N. Sahu, Phys. Rev. D 79, 103534 (2009) arXiv:0811.1866 [hep-ph]].

[39] V. S. Rychkov and A. Strumia, Phys. Rev. D 75, 075011 (2007) hep-ph/0701104.

[40] J. Edsjo and P. Gondolo, Phys. Rev. D 56, 1879 (1997) hep-ph/9704361. (see Eqs. (46-62).

[41] R. J. Scherrer and M. S. Turner, Phys. Rev. D 33, 1585 (1986) [Erratum-ibid. D 34, 3263 (1986)].

[42] R. Allahverdi, B. A. Campbell and J. R. Ellis, Nucl. Phys. B 579, 355 (2000) hep-ph/0001122.

[43] A. Anisimov and M. Dine, Nucl. Phys. B 619, 729 (2001) hep-ph/0008058.

[44] A. Anisimov, Phys. Atom. Nucl. 67, 640 (2004) [Yad. Fiz. 67, 660 (2004)] hep-ph/0111233.

[45] E. W. Kolb and M. S. Turner, "The Early Universe", Front. Phys. 69, 1 (1990).

[46] I. Affleck and M. Dine, Nucl. Phys. B 249, 361 (1985).

[47] K. A. Olive and M. Peloso, Phys. Rev. D 74, 103514 (2006) hep-ph/0608096.

[48] R. H. Cyburt, J. Ellis, B. D. Fields, F. Luo, 
K. A. Olive and V. C. Spanos, JCAP 0910, 021 (2009) arXiv:0907.5003 [astro-ph.CO]].

[49] M. Dine, L. Randall and S. D. Thomas, Phys. Rev. Lett. 75, 398 (1995) hep-ph/9503303.

[50] M. K. Gaillard, H. Murayama and K. A. Olive, Phys. Lett. B 355, 71 (1995) hep-ph/9504307.

[51] B. A. Campbell, M. K. Gaillard, H. Murayama and K. A. Olive, Nucl. Phys. B 538, 351 (1999) hep-ph/9805300.

[52] M. Dine and A. Kusenko, Rev. Mod. Phys. 76, 1 (2003) hep-ph/0303065.

[53] M. Kawasaki, K. Kohri, T. Moroi and A. Yotsuyanagi, Phys. Rev. D 78, 065011 (2008) arXiv:0804.3745 [hep$\mathrm{ph}]$.

[54] R. Allahverdi, R. H. A. D. Shaw and B. A. Campbell, Phys. Lett. B 473, 246 (2000) hep-ph/9909256.

[55] M. Postma and A. Mazumdar, JCAP 0401, 005 (2004) hep-ph/0304246.

[56] R. Allahverdi and A. Mazumdar, JCAP 0708, 023 (2007) hep-ph/0608296.

[57] A. Basboll, D. Maybury, F. Riva and S. M. West, Phys. Rev. D 76, 065005 (2007) hep-ph/0703015.

[58] A. Basboll, Phys. Rev. D 78, 023528 (2008) arXiv:0801.0745 [hep-th]].

[59] R. Allahverdi and A. Mazumdar, Phys. Rev. D 78, 043511 (2008) arXiv:0802.4430 [hep-ph]].

[60] A. E. Gumrukcuoglu, K. A. Olive, M. Peloso and M. Sexton, Phys. Rev. D 78, 063512 (2008) arXiv:0805.0273 [hep-ph]].

[61] A. E. Gumrukcuoglu, Phys. Rev. D 80, 123520 (2009) arXiv:0910.0854 [hep-ph]].

[62] R. Allahverdi, R. Brandenberger, F. -Y. Cyr-Racine and A. Mazumdar, Ann. Rev. Nucl. Part. Sci. 60, 27 (2010) arXiv:1001.2600 [hep-th]].

[63] A. Kusenko and M. E. Shaposhnikov, Phys. Lett. B 418, 46 (1998) hep-ph/9709492.

[64] K. Enqvist and J. McDonald, Phys. Lett. B 425, 309 (1998) hep-ph/9711514.

[65] S. Kasuya and M. Kawasaki, Phys. Rev. D 61, 041301 (2000) hep-ph/9909509.

[66] K. Enqvist, A. Jokinen and J. McDonald, Phys. Lett. B 483, 191 (2000) hep-ph/0004050.

[67] S. Kasuya and M. Kawasaki, Phys. Rev. Lett. 85, 2677 (2000) hep-ph/0006128.

[68] S. Kasuya and M. Kawasaki, Phys. Rev. D 62, 023512 (2000) hep-ph/0002285.

[69] S. Kasuya and M. Kawasaki, Phys. Rev. D 64, 123515 (2001) hep-ph/0106119.

[70] K. Enqvist, A. Jokinen, T. Multamaki and I. Vilja, Phys. Rev. D 63, 083501 (2001) hep-ph/0011134.

[71] T. Multamaki and I. Vilja, Phys. Lett. B 535, 170 (2002) hep-ph/0203195.

[72] K. Enqvist and J. McDonald, Nucl. Phys. B 570, 407 (2000) hep-ph/9908316. 